\title{
Reactivation of a suspended protocol
}

The hammer came down harder than Les Redmond had expected. He knew he was in trouble for being noncompliant with his IACUC protocol but for a change-and a change it would be-his noncompliance was inadvertent. Redmond had a poor track record with the Great Eastern University IACUC, having received IACUC-imposed sanctions twice before for protocol noncompliance incidents. However, neither of those sanctions included a protocol suspension. This time the IACUC wasn't interested in whether or not the problem was intentional; Redmond's protocol was suspended at a meeting of the full committee until the committee felt assured that the current and similar problems would not recur.

No matter how much Redmond ranted, threatened legal action against the IACUC and protested to the Institutional Official, the IACUC would not retreat from its position. Finally, Redmond gave up and asked Larry Covelli, the IACUC chairman, what he would have to do to get his protocol reinstated. "You have to understand," said Covelli, "the ball is in your court. You have to develop a plan to bring your research back into compliance and convince the IACUC that it will not see another noncompliance problem in the future. That's when a final decision will be made."

Redmond developed a plan that he thought would meet the demands of the IACUC. He presented it to the IACUC office and asked how long it would take before a decision was made. "I really don't know," said the IACUC's secretary, "but I'll ask Dr. Covelli and get back to you this afternoon."
After Redmond left, the secretary called Covelli and said, "Larry, every time the IACUC has reactivated a protocol, it just seems to happen, but I really don't know how it happens. What should I tell Dr. Redmond?"

Covelli replied that he would review Redmond's plan, and if it seemed adequate, he would send it to the IACUC by e-mail. If there were no questions or dissents, he would approve reactivating the protocol. However, if there were any questions or dissents, the decision would have to wait until the next full committee meeting so that Redmond's plan could be discussed and subsequently put to a formal committee vote.

Is the process described by Covelli appropriate to reactivate a suspended protocol?

\section{RESPONSE}

\section{Plan ahead for reactivation}

\section{Betty R. Theriault, DVM, DACLAM}

The appropriate process for IACUC suspension of protocol activities is well described in both the Animal Welfare Act and Animal Welfare Regulations (AWARs) ${ }^{1}$ and the Public Health Service Policy on Humane Care and Use of Laboratory Animals $(\text { PHS Policy })^{2}$. Although these documents do not specifically address reactivation of a suspended protocol, one can extract guidance on the steps the IACUC must take for protocol reactivation by examining the process for initial suspension.

Owing to the inherent gravity of a decision to suspend a protocol given the types of non-compliance that may lead to protocol suspension, both the AWARs and PHS Policy require that protocol suspensions be reported. Reporting to APHIS is required when the suspended activities involve
USDA-covered species, and reporting to OLAW is required if the protocol covers federally funded research. Additionally, both regulations require that any federal funding agencies financially supporting the research also be notified. At the time of reporting, it is expected that the Institutional Official (IO), in consultation with the IACUC, has reviewed the reason for suspension, that appropriate corrective action has been prescribed and that there are follow-up measures in place to ensure these corrective actions are sufficient and completed.

It appears that the IACUC of Great Eastern University has fallen short of its oversight obligations. It appears as though the IACUC did not discuss and agree upon a plan of corrective action or requirements for reactivation when it voted to suspend Redmond's protocol. Appropriate requirements could include training sessions for the principal investigator (PI), documentation of protocol compliance by the PI and his staff, a plan to ensure future compliance and possibly protocol amendments to address any unapproved or inadequately described activities in which the PI was engaged. At the time of protocol suspension, the IACUC should have discussed these measures and agreed that the PI submit all requested information and documentation before a scheduled convened quorum meeting for review and further action. At this meeting, the IACUC would have the opportunity to review protocol amendments (if indicated), training activities and documents from the PI and to then decide whether these measures satisfy the IACUC's requirements for protocol reactivation.

It is unclear from the scenario whether the protocol suspension was reported to Great Eastern's Institutional Official (IO) and to what extent the IO was involved in the corrective action process.

Without a plan of corrective action, the IACUC lacks defined expectations for the PI's actions, for the time line for completion of these actions and for how it will review and evaluate the corrective actions. This 